Available online on 15.11.2016 at http://jddtonline.info
Journal of Drug Delivery and Therapeutics
O 2016, publisher and licensee JDDT, This is an Open Access article which permits unrestricted
noncommercial use, provided the original work is properly cited

Review Article

\title{
THE MOLECULAR TRIAD SYSTEM INVOLVING RANK/RANKL/OPG AS THERAPEUTIC TARGETS FOR METABOLIC BONE DISEASES
}

\author{
Mekonnen Sisay ${ }^{1 *}$, Jemal Abdella $^{1}$, Yalew Molla ${ }^{2}$ \\ Eastern Ethiopia. \\ ${ }^{2}$ Department of Pharmacology, College of Health Sciences, Debre Markos University, Gojam, Ethiopia
}

${ }^{1}$ Department of pharmacology and Toxicology, School of Pharmacy, College of Health and Medical Sciences, Haramaya University, Harar,

\section{ABSTRACT}

Introduction: Metabolic bone diseases are disorders of bone strength, usually caused by abnormalities of minerals (such as calcium or phosphorus), vitamin $\mathrm{D}$, bone mass and/or structure. The most common metabolic bone disease is osteoporosis. It is a progressive bone disease that is characterized by a decrease in bone mass and density which can lead to an increased risk of fracture. This review was aimed to summarize a plenty of literatures related to the impact of Receptor Activator of Nuclear factor k-B (RANK)/RANK Ligand/Osteoprotegrin (OPG) system on bone metabolic diseases and therapeutic agents targeting this system.

Method: Data were collected from several legitimate data bases and services such as Pubmed, Pub med central, Medline, Hinari, Scopus, and other data base sources like Crossref, and Google scholar with the help of key words (RANK/RANKL/OPG system, metabolic bone diseases etc.). The data collection was carried out from July to October, 2016. Important data on the topic of interest were filtered properly. Review body: The role of RANK/RANKL/OPG system is well characterized within bone, where RANKL-RANK signaling mediates osteoclastogenesis, osteoclast activation and bone resorption via paracrine signaling between osteoblast and osteoclast cells. OPG produced by osteoblast and stromal cells in bone acts as a natural RANKL antagonist (decoy receptor) that intereferes with RANKL-RANK binding and hence prevents osteoclast differentiation and activation. This system and its interaction with various cytokines and calciotropic hormones in the regulation of osteoclastogenesis has led to a new era for further understanding of the pathophysiology of several disorders of bone metabolism including osteoporosis, primary bone tumors and rheumatoid arthritis. The system has also resulted in the recognition of several rare genetic disorders of bone mineral metabolism such as paget's disease, familial expansile osteolysis and osteopetrosis.

Conclusion: Since this cytokine system plays a major role in the pathogenesis of many disorders, several therapeutic agents targeting this system are being developed. Among them, denosumab, a monoclonal antibody against RANKL, is clinically approved for the treatment of osteoporosis and cancer-related bone diseases.

Key words: RANK/RANKL/OPG system, metabolic bone diseases, potential therapeutic approaches.

\footnotetext{
*Corresponding author

Mekonnen Sisay (BPharm, MSc in Pharmacology), Department of Pharmacology and Toxicology, School of Pharmacy, College of Health and Medical Sciences, Haramaya University, Harar, P.O.Box 235, Ethiopia,

Mail ID: mekonnensisay27@yahoo.com Cell phone: +251920-21-21-35

Article Info

Received 01 Oct 2016; Review Completed 17 Oct 2016; Accepted 28 Oct 2016, Available online 15 Nov 2016

Cite this article as: Sisay M, Abdella J, Molla Y, The molecular triad system involving RANK/RANKL/OPG as therapeutic targets for metabolic bone diseases, Journal of Drug Delivery \& Therapeutics. 2016; 6(60:31-39
}

DOI: http://dx.doi.org/10.22270/jddt.v6i6.1341 URI: http://iddtonline.info/index.php/iddt/article/view/1341

Declarations

Competing interests: The authors declared that there is no competing interest

Author contributions: MS collected literatures and reviewed it critically; JA and YM edited the literature review; MS prepared the manuscript for publication. All authors read and approved the final version of the manuscript.

Funding: None 


\section{INTRODUCTION}

Bone metabolism comprises the processes of bone formation and bone resorption, the key actions by which skeletal mass, structure and quality are accrued and maintained throughout life. In the mature skeleton, anabolic and catabolic actions are mostly balanced due to the tight regulation of the activity of bone forming cells (osteoblasts) and bone resorbing cells (osteoclasts) through circulating osteotropic hormones and locally active cytokines. ${ }^{1}$

Osteoblasts are derived from mesenchymal stem cells, and their differentiation is induced by several specific transcription factors, including core-binding factor $\alpha 1$ and osterix as well as by the bone morphogenic proteins (BMP-2, BMP-4, and BMP-7). Mature osteoblasts synthesize and secrete type I collagen, the major structural protein in bone matrix. Osteoblasts also secrete non-collagen proteins including osteocalcin, osteopontin, and bone sialoprotein, as well as cytokines including insulin like growth factor-1 (IGF-1), IL-1, and IL-6. 1,2 Osteoclasts are hematopoietic in origin and derive from granulocyte-macrophage progenitor cells. Osteoclastogenesis requires stimulation by macrophagecolony stimulating factor (M-CSF) and the binding of RANKL, expressed on osteoblasts, to RANK receptor on the osteoclast precursors. Osteoclast recruitment and activity are each highly regulated by cells of the osteoblast lineage. ${ }^{3,4}$

\section{METABOLIC BONE DISEASES}

Metabolic bone diseases (MBD) are disorders of bone strength, usually caused by abnormalities of minerals (such as calcium or phosphorus), vitamin D, bone mass and/or structure. MBD includes several bone diseases including osteoporosis, primary bone tumors, rare genetic disorders and inflammatory bone diseases (Table 1). The most common MBD is osteoporosis which is a progressive bone disease characterized by a decrease in bone mass and density which can in turn lead to an increased risk of fracture. ${ }^{5,6}$ In osteoporosis, the bone mineral density (BMD) is reduced, bone micro architecture deteriorates, and the amount and variety of proteins in bone are altered. It is defined by the World Health Organization (WHO) as a bone mineral density of 2.5 standard deviations or more below the mean peak bone mass (average of young, healthy adults) as measured by dual-energy X-ray absorptiometry. The disease may be classified as primary type 1, primary type 2, or secondary. The form of osteoporosis most common in women after menopause is referred to as primary type 1 or postmenopausal osteoporosis. Primary type 2 osteoporosis or senile osteoporosis occurs after age 75 and is seen in both females and males at a ratio of 2:1. Secondary osteoporosis may arise at any age and affect men and women equally. This form results from chronic predisposing medical problems or disease, or prolonged use of drugs such as glucocorticoids, where the disease is called glucocorticoid-induced osteoporosis. ${ }^{6-9}$

Osteoporosis is a major and growing public health problem in developed nations. Many women (30-50\%) and men (15-30\%) suffer a fracture related to it. Characteristic sites of fracture include vertebral bodies, the distal radius, and the proximal femur, but osteoporotic individuals have generalized skeletal fragility, and fractures at sites such as ribs and long bones also are common. ${ }^{6,10}$

Coming to primary bone tumors, multiple myeloma is the most common primary malignant bone tumor but is often considered a marrow cell tumor within the bone rather than a bone tumor. Osteosarcoma (osteogenic sarcoma) is the 2nd most common primary bone tumor and is highly malignant. It is most common among people aged 10 to 25 years, although it can occur at any age. Osteosarcoma produces malignant osteoid (immature bone) from tumor bone cells. Malignant giant cell tumor, which is rare, is usually located at the extreme end of a long bone. 7,11

Regarding genetic disorders of bone metabolism, Paget's disease is characterized by single or multiple sites of disordered bone remodeling. The etiology of the disease is uncertain but is thought to be the result of infection with the measles virus of the paramyxovirus family. It affects up to $2-3 \%$ of the population $>60$ years of age. The primary pathologic abnormality is increased bone resorption followed by exuberant bone formation. ${ }^{12}$ Osteopetrosis is also a rare polygenic disorder of generalized increased bone mass due to decreased osteoclastogenesis and bone resorption. ${ }^{13}$

Table 1: Summary of metabolic bone diseases

\begin{tabular}{|l|l|c|}
\hline Metabolic bone diseases & Sub classes & References \\
\hline \multirow{5}{*}{ Osteoporosis } & Postmenopausal osteoporosis & \multirow{2}{*}{$5-10$} \\
\cline { 2 - 2 } & Drug-induced osteoporosis & \\
\cline { 2 - 2 } & Immobilization-induced osteoporosis & \\
\cline { 2 - 2 } & Hyperparathyroidism induced osteoporosis & \\
\hline Primary malignant bone tumors & Osteo-sarcoma & \\
\cline { 2 - 2 } & Multiple myeloma & \\
\cline { 2 - 3 } & Giant cell tumor of the bone & \\
\hline Rare genetic disorders & Juvenile Paget's disease & \\
\cline { 2 - 3 } & Expansile skeletal hyperphosphatasia & $5-7$ \\
\cline { 2 - 3 } & Familial expansile osteolysis & \\
\cline { 2 - 3 } & Osteopetrosis & \\
\hline
\end{tabular}




\section{METHOD}

Data were collected from several legitimate data base sources and services such as Pubmed, Pub med central, Medline, Hinari, Scopus, and other data bases like Cross-ref, Socolar and Google scholar with the help of key words such as RANK/RANKL/OPG triad system, bone metabolism and associated disorders linked to this system as well as potential therapeutic approaches under study. Important data related to the topic of interest were filtered accordingly. Time frame was also adjusted to capture all valuable information regarding the impact of RANK/RANKL/OPG system on several MBDs as well as current potential therapeutic agents targeting this signaling pathway using different search engines. This review was conducted from July 2016 to October 2016 G.C.

The role of RANK/RANKL/OPG molecular triad system at glance

The RANK/RANKL/OPG molecular triad system has been shown to influence several biological and pathophysiological processes throughout the body including bone metabolism, immune system, mammary gland development and cancer. ${ }^{14,15}$ RANK/RANKL/ OPG molecular signaling plays an important role in lymph node development, lymphocyte differentiation, dendritic cell survival, T-cell activation, and tolerance induction (Figure 1). The RANKL/RANK axis also have direct osteoclast-independent effects on tumor cell development. ${ }^{16}$ RANK has also been shown to play a role in the thermoregulation signaling in females, which seems to be regulated by ovarian sex hormones. This key role of the RANK-RANKL system may link the osteoporosis and hot flashes seen as symptoms of hormonal changes in post-menopausal women. 17,18 OPG-deficient mice exhibited medial calcification of the aorta and a renal artery suggesting that regulation of OPG, and its signaling pathway play a role in vascular calcification and is a risk factor for development of atherosclerosis. ${ }^{19}$ A study conducted in diabetic Charcot neuropathy (DCN) patients showed that RANK/ RANKL/OPG signaling was involved in patients with DCN and played a role in vascular calcification. ${ }^{20}$

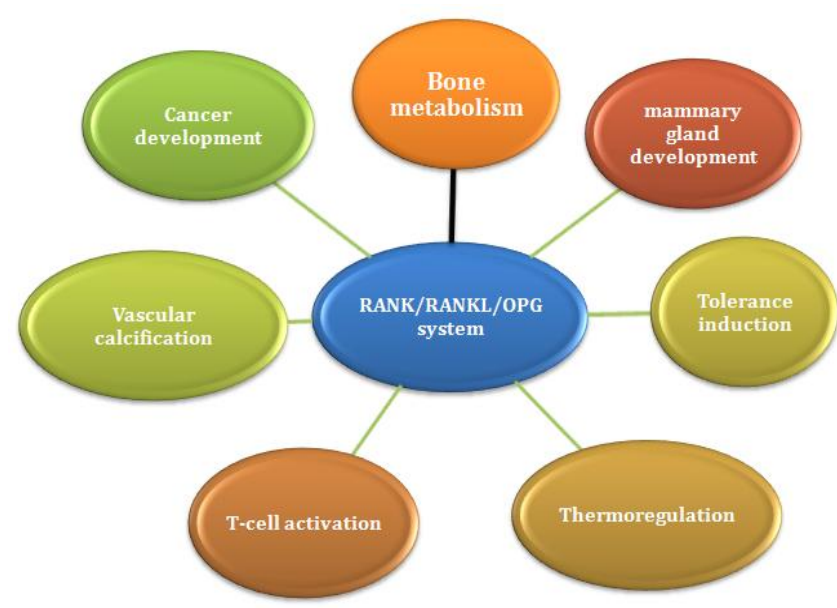

Figure 1: The role of RANK/RANKL/OPG system at glance
The role of RANK/RANKL/OPG molecular triad cytokine system is well characterized within bone, where RANKL/RANK signaling mediates osteoclastogenesis and bone resorption and OPG prevents RANK ligand interaction with RANK and hence prevent osteoclast differentiation and activation (Figure 2). ${ }^{21}$ RANKL induces the differentiation of osteoclast precursor cells and stimulates the resorption process and survival of mature osteoclasts. The TRAF adaptor proteins play an important role in the initial event of the signal transduction pathway induced by RANK. TRAFs $1,2,3,5$ and 6 are known to bind through the conserved TRAF domain to RANK. ${ }^{22,23}$

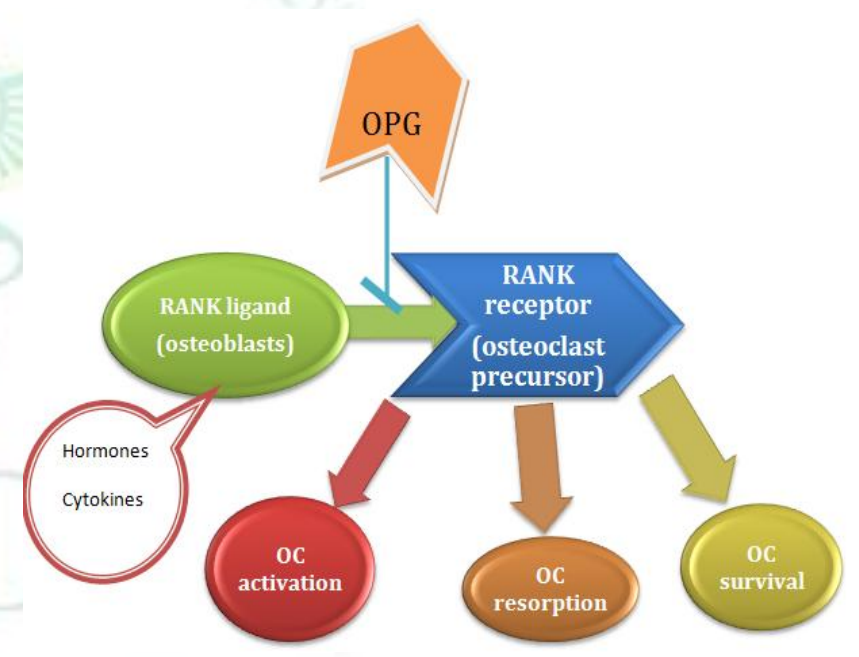

Figure 2: overview of RANK/RANKL/ OPG system in bone metabolism ( $\mathrm{OC}=$ osteoclast $)$

The RANK/RANKL/OPG system in metabolic bone diseases

The discovery of the RANK/RANKL/OPG system and its interaction with various cytokines and calciotropic hormones in the regulation of osteoclastogenesis has led to a new era of the pathophysiology of several disorders of bone metabolism. ${ }^{24}$ An imbalance of RANKL and OPG with an enhanced RANKL/OPG ratio favors osteoclast differentiation and activation and promotes bone loss. Systemic factors that enhance the RANKL/OPG ratio include glucocorticoids, immunosuppressants, estrogen deficiency, and continuous exposure to PTH. By contrast, the RANKL/OPG ratio is reduced by estrogens (17 $\beta$ estradiol, raloxifene, genistein) and mechanical strain. ${ }^{25}$

It became evident that many clinically relevant metabolic bone diseases in humans, including inflammatory bone diseases (e.g., rheumatoid arthritis), malignant bone tumors (e.g., myeloma or osteolytic metastases) and different forms of osteoporosis are related to alterations of the RANK/RANKL/OPG system. This system is also known to be involved in rare genetic disorders of bone metabolism, such as paget's disease and familial expansile osteoporosis. ${ }^{25,26}$

\section{Osteoporosis}

Estrogens are known to regulate various cytokines in the bone microenvironment that regulate OC function. 
Among which the RANK/RANKL/OPG system plays a significant role in estrogen induced bone remodeling. ${ }^{27}$

Estrogen deficiency causes both the early and late forms of osteoporosis in postmenopausal women. Since the discovery of RANK/RANKL/OPG system as a final pathway in osteoclast formation and differentiation, studies have confirmed the key role of this pathway in the pathogenesis of postmenopausal osteoporosis. In vitro experiments in human osteoblastic cell lines have shown a dose- and time-dependent increase in OPG mRNA in response to $17 \beta$-estradiol. Up-regulation of OPG is expected to decrease the interaction of RANKL with RANK in vivo and, hence, reduce osteoclastic bone resorption. Furthermore, the administration of OPG to ovariectomized rats was shown to prevent bone loss. ${ }^{28}$

Osteoblastic cells express both estrogen receptors, ER- $\alpha$ and ER- $\beta$. 17 $\beta$-estradiol increased OPG gene expression and protein secretion through a transcriptional mechanism in mesenchymal cells and mature osteoblastic cell lines that had been stably transfected with the ER- $\alpha$, while no such effect was observed in cells transfected with the ER- $\beta$. Phytoestrogens such as genistein are also able to enhance osteoblastic OPG production through ER $\alpha$-dependent mechanisms and concurrently suppress RANKL gene expression which is associated with an inhibition of osteoclastogenesis. $^{29,30}$ Another mechanism how estrogens interact with the OPG/RANKL/RANK system is associated with $17 \beta$-estradiol iduced repression of JNK activity, a critical down-stream signal of RANK pathway. This may attenuate the responsiveness of osteoclastic RANK receptors, thus diminishing the biological effects of RANKL. ${ }^{31}$

Long term systemic glucocorticoid administration results in rapid bone loss due to a combined effect of decreased bone formation and enhanced bone resorption. While increased osteoblastic apoptosis and decreased osteoblastic synthesis of bone matrix proteins largely account for decreased osteoblastic activity, an enhanced RANKL/OPG ratio may be a crucial paracrine mechanism for increased bone resorption. ${ }^{32}$ Glucocorticoids were found to suppress OPG mRNA expression and protein secretion and up-regulate RANKL mRNA expression in various osteoblastic cell models independently of the stage of differentiation and the baseline level of expression. Glucocorticoidresponse elements have been detected in the mouse RANKL gene, suggesting a regulatory mechanism through enhanced transcriptional activity. ${ }^{33,}{ }^{34}$ Studies of patients treated with glucocorticoids for both Crohn's disease and chronic glomerulonephritis demonstrated a significant increase in the serum RANKL/OPG ratio. This change correlated with increased levels of urinary and serum markers of bone resorption and with a fall in
BMD. $^{35,} 36$ Another study demonstrated higher OPG serum levels in patients with Cushing's syndrome compared to healthy controls, and OPG serum levels were significantly correlated with serum cortisol level. This indicates a difference between exogenous and endogenous glucocorticoid exposure or may be a mechanism to compensate bone loss in long-standing high cortisol levels. ${ }^{37}$

Immunosuppressants that are commonly used after allogeneic transplantation have been implicated in the pathogenesis of transplantation-associated osteoporosis. Different immunosuppressive agents concurrently enhance RANKL mRNA expression and suppress OPG mRNA levels and OPG protein secretion in undifferentiated human mesenchymal bone marrow stromal cells which appear to be rather susceptible to the effects of these drugs. By contrast, mature osteoblasts are relatively resistant against these drugs. ${ }^{38}$

RANK/RANKL/OPG system has implicated as a paracrine mediator of mechanical strain on bone metabolism. Mechanical strain applied to murine primary stromal cells decreased RANKL mRNA levels by approximately $40 \%$ which was paralleled by a $50 \%$ reduction of osteoclast formation. More detailed followup studies indicated that RANKL inhibition following mechanical strain critically depends on the activation of the extracellular regulated kinase (ERK) 1/2 pathway. ${ }^{39}$. ${ }^{40}$ Blockade of RANKL using parenteral administration of OPG has been used to reduce bone loss associated with immobilization in sciatic nerve damage and tailsuspension experiments. In a murine model of sciatic nerve crush, OPG administration reduced femoral bone loss in a dose-dependent manner. Similarly, in the murine tail suspension model, OPG treatment mitigated bone loss by decreasing femoral endocortical resorption by $24 \% .{ }^{41,42}$

Continuously increased and unregulated serum PTH concentrations, as presented in hyperparathyroidism, resulted in increased bone resorption and bone loss. In vitro, PTH was found to stimulate RANKL mRNA levels and to decrease OPG production in osteoblastic cells and to increase the RANKL/OPG ratio. All of these changes were associated with increased osteoclast formation. ${ }^{43}$ The potential molecular basis for the dual clinical effects of PTH, catabolic effect on continuous exposure and anabolic effect on intermittent exposure, was suggested by an elegant in vitro model. While continuous PTH exposure enhanced the RANKL/OPG ratio by up to 25 -fold and stimulated osteoclastogenesis, intermittent PTH exposure stimulated IGF-1 mRNA, an anabolic skeletal growth factor. What is more, treatment of rats with PTH also decreased OPG mRNA levels in rat femur metaphyseal and diaphyseal bone in vivo. ${ }^{44,45}$ 
Table 2: Summary of osteoporosis types linked with RANK/RANKL/OPG system

\begin{tabular}{|c|c|c|}
\hline Types of osteoporosis & Linkage to RANK/RANKL/OPG system & Ref. \\
\hline \multirow[t]{4}{*}{$\begin{array}{l}\text { Postmenoposal } \\
\text { osteoporosis }\end{array}$} & $\begin{array}{l}\text { In vitro experiments in human osteoblastic cell lines have shown a dose- and time- } \\
\text { dependent increase in OPG mRNA in response to } 17 \beta \text {-estradiol }\end{array}$ & \\
\hline & Administration of OPG to ovariectomized rats was shown to prevent bone loss & 28 \\
\hline & $\begin{array}{l}\text { 17ß-estradiol iduced repression of JNK activity, a critical down-stream signal of } \\
\text { RANK pathway }\end{array}$ & \\
\hline & Phytoestrogens are also able to enhance osteoblastic OPG production & 29,30 \\
\hline \multirow{3}{*}{$\begin{array}{l}\text { Drug induced } \\
\text { osteoporosis }\end{array}$} & Glucocorticoids were found to suppress OPG mRNA expression & 33,34 \\
\hline & Glucocorticoids increase in the serum RANKL/OPG ratio & 35,36 \\
\hline & $\begin{array}{l}\text { immunosuppressive agents concurrently enhance RANKL mRNA expression and } \\
\text { suppress OPG mRNA levels }\end{array}$ & 38 \\
\hline \multirow[t]{2}{*}{$\begin{array}{l}\text { Immobilization induced } \\
\text { osteoporosis }\end{array}$} & $\begin{array}{l}\text { In a murine model of sciatic nerve crush, OPG administration reduced femoral } \\
\text { bone loss in a dose-dependent manner }\end{array}$ & 41 \\
\hline & $\begin{array}{l}\text { In the murine tail suspension model, OPG treatment mitigated bone loss by } \\
\text { decreasing femoral endocortical resorption }\end{array}$ & 42 \\
\hline \multirow[t]{2}{*}{$\begin{array}{l}\text { Hyperthyroidism } \\
\text { induced osteoporosis }\end{array}$} & $\begin{array}{l}\text { In vitro, PTH was found to stimulate RANKL mRNA levels and to decrease OPG } \\
\text { production in osteoblasts (increase the RANKL/OPG ratio) }\end{array}$ & \\
\hline & PTH also decreased OPG mRNA levels & 44 \\
\hline
\end{tabular}

\section{Rheumatoid arthritis}

Rheumatoid arthritis (RA) is a chronic inflammatory condition associated with progressive destruction of the articular synovial lining. T cell invasion of the synovium plays a crucial role in the obliteration of the articular cartilage and adjacent bone. Furthermore, fibroblast-like synovial cells have been shown to play a role in this process. It has been demonstrated that $\mathrm{T}$ cells express RANKL and that there is over-expression of RANKL mRNA in the synovium of RA patients at the site of bone resorption Elevated serum levels of soluble RANKL and OPG have been demonstrated in patients with RA, and these factors normalize after treatment with anti-TNF therapy. ${ }^{46,47}$ A recent study showed that Stromal cellderived factor 1 (SDF-1) is a chemokine that is involved in the bone-destructive process in RA and bony metastasis in malignancy. It induces osteoclastogenesis directly and indirectly via up-regulating RANKL expression in RA synovial fibroblasts and CD4+ T cells, and that this is mediated by TNF $\alpha$. The axis of SDF-1 and RANKL is a potential therapeutic target for RAassociated bone destruction. ${ }^{48}$

\section{Primary malignant bone tumors}

Osteosarcoma, the most frequent primary malignant bone tumor, is defined as a malignant tumor originates from mesenchymal cells characterized by the direct malignant osteoid and/or woven bone formation by tumor cells. ${ }^{49}$ It is strongly associated with abnormal RANKL/RANK/ OPG balance that leads to pathological bone fractures. Grimaud et al. demonstrated an increase in serum RANKL/OPG ratio in the patients with high grade osteosarcoma. ${ }^{50}$ OPG treatment achieved not only the prevention of osteosarcoma-induced osteolysis but also the inhibition of associated tumor development that improved survival rate in OPG-treated animals. The study failed to demonstrate direct effect of OPG on tumor cells survival since it also blocks TNF related apoptosis inducing ligand (TRAIL). ${ }^{51}$
Multiple myeloma (MM) is a plasma cell malignancy that develops in the bone marrow and characterized by the development of osteolytic bone lesions associated with bone pain, hypercalcemia and pathological fractures. Three cell types are involved in this pathology:- myeloma cells, bone marrow stromal cells and osteoclasts. Imbalance in the RANK/RANKL/OPG system was suggested as an alternative mechanism for enhanced osteoclastogenesis in bone loss associated with multiple myeloma. Derangements in the RANK/RANKL/OPG system may be due to alterations in RANKL production and/or increased lysosomal degradation of OPG. Increased RANKL expression in multiple myeloma may be due to its direct production by myeloma cells or its indirect production through the increased synthesis of IL7 , which then over express RANKL in stromal cells. ${ }^{52}$

Giant cell tumor of bone (GCTB) is an osteolytic, usually benign neoplasm characterized by infiltration with osteoclast-like giant cells, and the osteoclast differentiation factor, RANKL is heavily involved in its pathogenesis. The stromal cells within GCTB samples had increased RANKL/OPG ratio compared to that of non-osteolytic bone tumors. ${ }^{53}$

\section{Rare genetic disorders}

Discovery of the RANK/RANKL/OPG system has led to recognition of several rare genetic disorders of bone mineral metabolism.

Mutations in the OPG gene can cause abnormalities in the ligand-binding properties of OPG, resulting in its inactivation and a disorder with diverse phenotypic presentations.

$\checkmark$ Juvenile Paget's disease (JPD) is a rare autosomal recessive disorder that presents in early childhood with bone deformities, fractures, hearing deficits, and dental abnormalities of variable severity. The disorder can be due to an inactivating mutation in the OPG gene (TNFRSF11B), localized to chromosome 8q24.2. Missense mutations in the cysteine residues of 
OPG are predicted to interfere with its ligand-binding domain and are responsible for the most severe phenotype of JPD. In more intermediate forms of JPD, missense mutations in residues other than cysteine are present in the ligand-binding region of the OPG. In addition, an insertion-deletion in exon 5 of this gene has been associated with a milder form of the disease. All of these mutations lead to unopposed activation of RANK to varying degrees, resulting in enhanced osteoclastogenesis and consequently increased bone turnover. $^{54}$

Mutations in the RANK gene (TNFRSF11A) that disrupt the signal peptide region of the protein result in the lack of normal cleavage of the signal peptide and an increase in RANK-mediated signaling. These activating mutations result in three different phenotypic presentations.

$\checkmark$ Early-onset Paget's disease of bone (PDB2) is a heterogeneous, autosomal dominant skeletal disorder characterized by bone deformities, hearing deficits, and dental problems. PDB2 is due to an activating mutation in the RANK gene comprised of a 27-bp tandem duplication. ${ }^{55}$

$\checkmark$ Expansile skeletal hyperphosphatasia, an autosomal dominant disorder that presents with early onset of deafness, dental defects, and accelerated bone turnover manifested by pain in the bones and episodic hypercalcemia. Skeletal symptoms begin before puberty and progress with episodes of exacerbation until middle age. The activating mutation in the RANK gene is caused by 15-bp tandem duplication. ${ }^{56}$

$\checkmark$ Familial expansile osteolysis (FEO) is an autosomal dominant disorder that presents in early childhood to young adulthood with hearing deficit. Osteopenia is a common feature. The major skeletal finding in this disorder is osteolysis followed by bony expansion due to fat deposition rather than osteosclerosis. The genetic abnormality is an activating mutation in the RANK gene linked to an 18-bp tandem duplication. ${ }^{57}$

Furthermore, mutations of the sequestosome 1 gene (SQSTM1), which encodes an important scaffold protein in the NF-kB pathway, are a common cause of classical PDB. The rare syndrome of hereditary inclusion body myopathy, PDB, and fronto-temporal dementia is caused by mutations in the valosin containing protein (VCP) gene. This gene encodes VCP, which has a role in targeting the $\operatorname{Ik} \beta$ (downstream molecule of this triad system) for degradation by the proteasome. ${ }^{58}$

\section{Osteopetrosis.}

RANK, RANKL, OPG, as well as their signaling pathway may play a potential role in the pathogenesis of osteopetrosis. For instance, mice deficient in RANKL, RANK have shown severe osteopetrosis. Moreover, transgenic mice over expressing either OPG or soluble RANK both of which neutralize RANKL also have osteopetrosis. $^{13,59}$

\section{POTENTIAL THERAPEUTIC APPROACHES}

\section{Monoclonal antibodies}

Denosumab is a fully human monoclonal antibody that avidly binds RANKL and blocks RANKL/RANK interaction and signaling. Unlike OPG, denosumab does not bind to TRAIL and hence considered to be beneficial for treatment of cancer metastasis to bone. Denosumab was approved by FDA in 2010 for the prevention of SREs from bone metastases in solid malignancies, as well as for the treatment of osteoporosis in postmenopausal women and men at high risk for fracture. This agent is administered subcutaneously with dose of $120 \mathrm{mg}$ every 4 weeks for the prevention of SREs, and with doses of 60 mg every 6 months for its osteoporosis and bone loss indications. The mean half-life of denosumab is approximately 28 days. $^{60,61}$ FDA approved denosumab, a new class of drugs that inhibit RANKL, for the treatment of patients whose GCTB is unresectable, or when surgery is likely to result in severe morbidity. ${ }^{53}$

\section{Osteoprotegerin like peptidomimetics}

Osteoprotegerin like peptidomimetics (e.g., OP3-4) are designed to block the RANKL/RANK interaction and to inhibit osteoclastic bone resorption. OP3-4 selectively inhibits RANKL but not TRAIL, and hence is effective in preventing malignant bone diseases. Refining the design of such peptides may improve efficacy and allow their use in both dissecting the relative importance of osteoprotegerin/RANKL interactions versus osteoprotegerin/TRAIL interactions. This may lead to improved therapeutic approaches for treating myeloma and the associated bone disease. ${ }^{62}$

\section{RANK receptor inhibitors}

Following identification of cytoplasmic motif of RANK involved in osteoclast differentiation, cell-permeable inhibitors termed the RANK receptor inhibitors (RRI), which target this motif were developed. The RRI peptide blocked RANKL-induced osteoclast formation from murine bone marrow derived macrophages. Furthermore, RRI inhibited the resorptive function of osteoclasts and induced osteoclast apoptosis. Treatment with the peptide impaired downstream signaling of RANK linked to Vav3, Rac1, and Cdc42 and resulted in disruptions of the actin cytoskeleton in differentiated osteoclasts. In addition, RRI blocked inflammation-induced bone destruction and protected against ovariectomy-induced bone loss in mice. These data may be useful in the development of selective therapeutic agents for the treatment of osteoporosis and other bone diseases. ${ }^{63}$

\section{Novel peptide RANK antagonists}

It is an alternative approach to designing small peptides that able to specifically bind to the hinge region of membrane RANK responsible for the conformational change upon RANKL association. A nona peptide generated by this method was validated for its biological activity in vitro and in vivo and served as a lead compound for the generation of a series of peptide RANK antagonists derived from the original sequence. This novel, effective and affordable small peptide inhibitors specifically targets the receptor RANK and opens a new therapeutic opportunity for the treatment of resorptive bone disease. ${ }^{64}$ 


\section{Ezymatic cleavage of RANK ectodomain}

Enteropeptidase can inhibit the RANKL-RANK signalling pathway through the cleavage of RANK. A surrogate peptide blocking assay indicated that enteropeptidase could specifically cleave RANK on the sequence NEEDK. Osteoclast differentiation assay and $\mathrm{NF}-\kappa \mathrm{B}$ activity assay confirmed that enteropeptidase could inhibit osteoclastogenesis in vitro through the cleavage of RANK. ${ }^{65}$

\section{Selective Small Molecules}

Modulation of OPG in the bone micro environment can be as effective as exogenously administered OPG-Fc in inhibiting the growth of tumor deposits in bone and the periarticular resorption of adjuvant arthritis. Small molecules that selectively stimulate endogenous OPG expression could have therapeutic benefits similar to those of native OPG in reducing both the formation and the activity of osteoclasts. This could provide a new approach to development of drugs for treatment of diseases of increased bone resorption, such as osteoporosis, Paget's disease, osteolytic bone metastases, hypercalcemia of malignancy, and rheumatoid arthritis. ${ }^{66}$

\section{RANKL targeted peptides}

A novel peptide was designed from OPG with dual inhibitory activity against bone loss and inflammation using site-directed mutagenesis. Out of the three putative sites (i.e., Tyr70-Asp78, Tyr82-Glu96, and Leu113Arg122) available on OPG for RANKL binding, Leu113Arg122 was used as a template for peptide synthesis. Peptide mutants of the template sequence were synthesized and initially screened for their inhibitory effect on RANK-RANKL binding by competitive ELISA. The study provided a basis about the therapeutic benefit of this novel peptide in the prevention of bone loss and inflammation in rheumatoid arthritis with reduced side effects. ${ }^{67}$

\section{RNA interference technology}

In the study conducted to assess the effect of inhibition of RANK expression in mouse bone marrow macrophages (BMM), three pairs of short hair pin RNAs (shRNA) targeting RANK were designed and synthesized. Among the three shRANKs examined, shRANK-3 significantly suppressed [88.3\%] the RANK expression. shRANK-3 also brought about a marked inhibition of osteoclast formation and bone resorption as demonstrated by tartrate resistant acid phosphatase (TRAP) staining and osteoclast resorption assay. The study showed that retrovirusmediated shRANK-3 suppresses osteoclast differentiation and osteolysis of BMMs in vitro. ${ }^{68}$

\section{Strontium ranelate}

Strontium ranelate is an oral daily drug formed of two atoms of stable strontium and an organic moiety (ranelic acid). Strontium ranelate combines the antiresorptive effect on bone with an additional anabolic action. In vitro studies have shown different effects of strontium ranelate on osteoblasts and osteoclasts. In the in vitro study conducted on osteoblasts, strontium ranelate induced a concentration-dependent increase in OPG mRNA expression as well as OPG secretion. On the other hand, RANKL mRNA and concentration were markedly decreased. The proposed mediator of these effects by Brennan et al was the calcium-sensing receptor. Indeed, knocking down the receptor abolished the effects of strontium ranelate on OPG and RANKL. ${ }^{69}$

\section{Chloroquine}

TRAF3 limits RANKL-induced osteoclastogenesis by suppressing canonical and non canonical NF- $\kappa \mathrm{B}$ signaling. Conditional osteoclast -specific TRAF3-Knock out (cKO) mice had mild osteoporosis and increased osteoclast formation. RANKL induced TRAF3 degradation via the lysosome/autophagy system. The autophagy/lysosome inhibitor chloroquine reduced RANKL-induced osteoclast formation and function by increasing TRAF3 expression in OCPs in vitro and in vivo. Although chloroquine had no effect on basal bone resorption, it inhibited parathyroid hormone and ovariectomy-induced osteoclast activation in wild type mice. $^{70}$

\section{Phytopharmaceuticals}

Jolkinolide B (JB) isolated from the root of Euphorbia fischeriana inhibited RANKL-induced OC differentiation from bone marrow macrophages (BMMs) without cytotoxicity. Furthermore, the expression of osteoclastic marker genes, such as tartrate-resistant acid phosphatase (TRAP), cathepsin K (CtsK), and calcitonin receptor (CTR), was significantly inhibited. JB inhibited RANKLinduced activation of NF- $\kappa \mathrm{B}$ by suppressing RANKLmediated $\mathrm{I} \kappa \mathrm{B} \alpha$ degradation. Moreover, JB inhibited RANKL-induced phosphorylation of mitogen-activated protein kinases (p38, JNK, and ERK). It is identified that $\mathrm{JB}$ as an inhibitor of osteoclast formation and provides evidence that $\mathrm{JB}$ might be an alternative medicine for preventing and treating osteolysis. ${ }^{71}$ Bone protective effect of water extract of the stem of Acer tegmentosum (WEAT) was also investigated. It was found that WEAT inhibits osteoclast differentiation induced by RANKL. In osteoclast precursor cells, WEAT inhibited RANKL-induced activation of JNK, $\mathrm{NF}-\kappa \mathrm{B}$, and cAMP response element-binding protein (CREB), leading to suppression of the induction of $\mathrm{c}$ Fos and NFATc1, key transcription factors for osteoclast differentiation. In addition, WEAT inhibited bone resorbing activity of mature osteoclasts. Furthermore, the oral administration of WEAT reduced RANKL-induced bone resorption and trabecular bone loss in mice in vivo. ${ }^{72}$

\section{CONCLUSION AND FUTURE PERSPECTIVES}

The role of RANK/RANKL/OPG system in metabolic bone disorders is well emphasized in this review. RANK/RANKL/OPG molecular triad can be a hot spot as a therapeutic device in the areas of bone metabolic diseases and oncology. This system has shown a significant contribution on the pathogenesis of various types of osteoporosis, primary malignant bone tumors, rare genetic disorders as well as arthritis. Bone degradation by osteoclasts plays a crucial role in bone tumor development and control of local osteoclast activity. This can provide a potential therapeutic strategy 
targeting the RANK/RANKL/OPG triad system via different pathways, mainly blocking vicious cycle which resides between bone degradation and growth of tumor cells. Such therapies may be used in combined setting with conventional therapeutic approaches. In general, the RANK/RANKL/OPG triad and its signaling hold a great promise for the treatment of bone metabolic disorders and malignant tumors. Further studies are needed to determine orchestrated effects of this system by inhibiting osteoclast activity, tumor migration and immunomodulation

\section{REFERENCES}

1. Offermanns S, Rosenthal W. Encyclopedia of Molecular Pharmacology. 2008. $2^{\text {nd }}$ ed. Spriger. PP 277-279.

2. Neve A, Corrado A, Cantatore FP., Osteoblast physiology in normal and pathological conditions, Cell and Tissue Research 2011; 343:289-302.

3. Roodman GD., Cell biology of the osteoclast, Experimental Hematology 1999; 27:1229-41.

4. Teitelbaum SL, Ross FP. Genetic regulation of osteoclast development and function, Natural Revolutionary Genetics 2003;4: 638-49

5. Charles RC, Robert ES. Modern Pharmacology with clinical applications. In : Schwartz FL. Parathyroid Hormone, Calcitonin, Vitamin D, and other compounds related to mineral metabolism. Chapter 66. 2010; $5^{\text {th }}$ ed. pp759-60

6. Laurence LB. Goodman \& Gilman's the pharmacological basis of therapeutics. In: Agents Affecting Mineral Ion Homeostasis and Bone Turnover. The McGraw-Hill Companies. 2010; $12^{\text {th }}$ ed. California.

7. Katzung BG. Basic and Clinical Pharmacology. In: Bikle DD. Agents that affect bone mineral homeostasis, chapter 12 , McGraw-Hill Companies. 2012; $12^{\text {th }}$ ed.PP 782

8. WHO. Assessment of fracture risks and its application to screening for postmenopausal osteoporosis, Report of a WHO Study Group, World Health Organization technical report series 1994;843: 1-129

9. Ginaldi L, Benedetto M C, Martinis M. Osteoporosis, inflammation and ageing, immunity \& Ageing 2005; $2: 14$

10. Teng GG, Curtis JR, Saag KG. Mortality and osteoporotic fractures: Is the link causal, and is it modifiable? Clin Exp Rheumatol, 2008, 26:S125-S137.

11. Joyce MJ. Primary malignant bone tumors. 2012; Available at:

http://www.merckmanuals.com/professional/musculoskeletaland-connective-tissue disorders/tumors-of-bones-and-joints

12. Roodman GD. Windle JJ. Paget disease of bone. J Clin Invest. 2005; 115:200-08.

13. Felix R, Cecchini MG, Hofstetter W., Recent developments on the understanding of the pathophysiology of osteopetrosis, European Journal of Endocrinology 2003; 134:143-56.

14. Boyce BF, Xing L. Biology of RANK, RANKL, and osteoprotegerin. Arthritis Res Ther. 2007;9(1):1.

15. Fata JE, Kong YY, Li J, Sasaki T, Irie-Sasaki J, Moorehead RA, et al. The osteoclast differentiation factor osteoprotegerin-ligand is essential for mammary gland development. Cell;103(1):41-50.

16. Cheng ML, Fong L. Effects of RANKL-targeted therapy in immunity and cancer. Front Oncol. 2014; 3:329. doi:10.3389/fonc.2013.00329.

17. Hanada R, Le ibbrand t A, Hanada T , Kitaoka S, Fu ruyas hiki $\mathrm{T}, \mathrm{Fu}$ jihara $\mathrm{H}$, et al. Central control of fever and female body temperature by RANKL/RANK. Nature. 2009; 462(7272):505- 9

18. Hanada R. The new function of RANKL/RANK system in the central nervous systems. Neurosci Res. 2011 Sep;71:e22.

19. Bucay N, Sarosi I, Dunstan CR, et al. osteoprotegerindeficient mice develop early onset osteoporosis and arterial calcification, genes and developmen 1998; 12: 1260-1268
20. Ndip A, Williams A, Edward B, et al., The RANKL/RANK/OPG Signaling Pathway Mediates Medial Arterial Calcification in Diabetic Charcot Neuroarthropathy, Diabetes Journal 2011; 60:2187 -2196

21. Hofbauer LC, Heufelder, Armin E. Role of receptor activator of nuclear factor- $\mathrm{\kappa B}$ ligand and osteoprotegerin in bone cell biology, Journal of Molecular Medicine 2001; 79:243-53

22. Galibert L,Tometsko ME, Anderson DM. The involvement of multiple tumor necrosis factor receptor (TNFR)-associated factors in the signaling mechanisms of receptor activator of NF-kappaB, a member of the TNFR superfamily, Jouranal of Biological Chemistry 1998; 273: 34120-7.

23. Wong BR, Rho J, Arron J. TRANCE is a novel ligand of the tumor necrosis factor receptor family that activates c-Jun Nterminal Kinase in $\mathrm{T}$ cells, Journal of Biological Chemistry $1997 ; 272: 25190-4$.

24. Dougall WC. Molecular Pathways: Osteoclast-Dependent and Osteoclast-Independent Roles of the RANKL/RANK/OPG Pathway in Tumorigenesis and Metastasis, Clinical Cancer Research 2011; 18:326-35

25. Hofbauer LC, Kühne CA, Viereck V. The OPG/RANKL/ RANK system in metabolic bone diseases, Journal of Musculoskeletal and Neuron Interaction 2004; 4:268-75

26. Teitelbaum SL., Bone resorption by osteoclast, Science 2000; 289:1504-08

27. Riggs BL. the mechanisms of estrogen regulation of bone resorption, journal of clinical investigation 2000; $106: 10$

28. Hofbauer LC, Khosla S, Dunstan CR, et al. Estrogen stimulates gene expression and protein production of osteoprotegerin in human osteoblastic cells, Endocrinology 1999; 140:4367-70.

29. Saika M, Inoue D, Kido S, et a1. $17 \beta$-Estradiol stimulates expression of osteoprotegerin by a mouse stromal cell line, ST-2, via estrogen receptor, Endocrinology 2001; 142:2205-12

30. Chen XW, Garner SC, Anderson JJ. Isoflavones regulate interleukin-6 and osteoprotegerin synthesis during osteoblast cell differentiation via an estrogen-receptor-dependent pathway, Biochemical and Biophysical Research Communication 2002; 295:417-22.

31. Shevde NK, Bendixen AC, Dienger KM. et al. Estrogens suppress RANK ligand-induced osteoclast differentiation via a stromal cell independent mechanism involving c-Jun repression, Proceeding national academy of sciences 2000; 97:7829-34

32. Hofbauer LC, Gori F, Riggs BL. Stimulation of osteoprotegerin ligand and inhibition of osteoprotegerin production by glucocorticoids in human osteoblastic lineage cells: potential paracrine mechanisms of glucocorticoid induced osteoporosis, Endocrinology 1999;140:4382-9.

33. Kitazawa R, Kitazawa S, Maeda S. Promoter structure of mouse RANKL/TRANCE/OPGL/ODF gene, Biochemical and Biophysical research communications 1999; 1445 :134-41

34. Vidal NOA, Brändström $\mathrm{H}$, Jonsson $\mathrm{KB}$, et al. Osteoprotegerin mRNA is expressed in primary human osteoblast-like cells: down-regulation by glucocorticoids, Journal of Endocrinology 1998; 159:191-5.

35. Sasaki N, Kusano E, Ando $Y$, et al. Changes in osteoprotegerin and markers of bone metabolism during glucocorticoid treatment in patients with chronic glomerulonephritis, Bone 2002; 30:853-58

36. Von-Tirpitz C, Epp S, Klaus J, et al. Effect of systemic glucocorticoid therapy on bone metabolism and the osteoprotegerin system in patients with active Crohn's disease, European Journal of Gastroenterology and Hepatology 2003; 15:1165-70

37. Ueland $\mathrm{T}$, Bollerslev $\mathrm{J}$, Godang $\mathrm{K}$, et al. Increased serum osteoprotegerin in disor-ders characterized by persistent immune activation or glucocorticoid excess - possible role in bone homeostasis, European Journal of Endocrinology 2001; 145:685-90.

38. Hofbauer LC, Shui C, Riggs BL, et al. Effects of immunosuppressants on receptor activator of NF-kB ligand and osteoprotegerin production by human osteoblastic and 
coronary artery smooth muscle cells, Biochemical and Biophysical Research Communication 2001; 280:334-9

39. Rubin J, Murphy T, Nanes MS, et al. Mechanical strain inhibits expression of osteoclast differentiation factor by murine stromal cells, American Journal of Physiology Cell Physiology 2000; 278:1126-32.

40. Rubin J, Murphy TC, Fan X, et al. Activation of extracellular signal-regulated kinase is involved in mechanical strain inhibition of RANKL expression in bone stromal cells, Jouranal of Bone Mineral Research 2002 17:1452-60

41. Bateman TA, Dunstan CR, Ferguson VL, et al. Osteoprotegerin mitigates tail suspension-induced osteopenia, Bone research 2000; 26:443-9.

42. Bateman TA, Dunstan CR, Lacey DL,et al. Osteoprotegerin ameliorates sciatic nerve crush induced bone loss, Journal of Orthopedic research 2001; 19:518-23.

43. Lee SK, Lorenzo JA. Parathyroid hormone stimulates TRANCE and inhibits osteoprotegerin messenger ribonucleic acid expression in murine bone marrow cultures: correlation with osteoclast-like cell formation, Endocrinology 1999; 140:3552-61

44. Onyia JE, Miles RR, Yang X, et al. In vivo demonstration that parathyroid hormone 1-38 inhibits the expression of osteoprotegerin in bone with the kinetics of an immediate early gene. Journal of Bone Mineral Research 2000; 15:86371

45. Locklin RM, Khosla S, Turner RT, et al. Mediators of the biphasic responses of bone to intermittent and continuously administered parathyroid hormone, Journal of Cellular Biochemistry 2003; 89:180-90.

46. Ziolkowska M, Kurowska M, Radzikowska A, et al. High levels of osteoprotegerin and soluble receptor activator of nuclear factor $\mathrm{\kappa B}$ ligand in serum of rheumatoid arthritis patients and their normalization after anti-tumor necrosis factor $\alpha$ treatment, Arthritis Rheumatology 2002; 46:1744-53

47. Abeles AM, Pillinger MH. the role of the synovial fibroblast in rheumatoid arthritis: cartilage destruction and the regulation of matrix metalloproteinases, Bulletin of the NYU Hospital for Joint Diseases, 2006;64:20-24

48. Kim HR, Kim KW, Kim BM, et al. Reciprocal activation of CD4+ T cells and synovial fibroblasts by stromal cell-derived factor 1 promotes RANKL expression and osteoclastogenesis in rheumatoid arthritis, 2014; 66:538-48

49. Herzog CE. Overview of sarcomas in the adolescent and young adult population, Journal of Pediatric Hematology and Oncology 2005; 27:215-8.

50. Grimaud E, Soubigou L, Couillaud S, et al. Receptor activator of nuclear factor kappaB ligand (RANKL)/osteoprotegerin (OPG) ratio is increased in severe osteolysis, Americal journal of Pathology 2003; 163:2021-31

51. Lamoureux F, Richard P, Wittrant Y, et al. Therapeutic relevance of osteoprotegerin gene therapy in osteosarcoma: blockade of the vicious cycle between tumor cell proliferation and bone resorption. Cancer Research 2003; 67:7308-18

52. Pearse RN, Sordillo EM, Yaccoby S, et al. Multiple myeloma disrupts the TRANCE/osteoprotegerin cytokine axis to trigger bone destruction and promote tumor progression, Proceeding National academy of sciences 2001; 98:11581-6

53. Lewin J, Thomas D. Denosumab: a new treatment option for giant cell tumor of bone, Drugs Today 2013; 49:693-700

54. Whyte MP, Obrecht SE, Finnegan PM, et al. Osteoprotegerin deficiency and juvenile Paget's disease, New England, Journal of Medicine 2002;347:175-84

55. Nakatsuka K, Nishizawa Y, Ralston SH. Phenotypic characterization of early onset Paget's disease of bone caused by a 27-bp duplication in the TNFRSF11A gene, Journal of Bone Mineral Research 2003;18:1381-5

56. Whyte MP, Hughes AE. Expansile skeletal hyperphosphatasia is caused by a15-base pair tandem duplication in TNFRSF11A encoding RANK and is allelic to familial expansile osteolysis, Journal of Bone Mineral Research 2002; 17:26-29

57. Whyte MP, Reinus WR, Podgornik MN, et al. Familial expansile osteolysis in 5 generation American kindred, Medicine (Baltimore) 2002; 81:101 -21

58. Daroszewska A and Ralston SH. Mechanisms of disease: genetics of Paget's disease of bone and related disorders, National Clinical Practice of Rheumatololgy 2006; 2:270-7

59. Iotsova V, Caamano J, Loy J, et al. Osteopetrosis in mice lacking $\mathrm{NF}-\kappa \mathrm{B} 1$ and $\mathrm{NF}-\kappa \mathrm{B} 2$, Natural Medicine 1998; 3:1285-9

60. Kostenuik PJ, Nguyen HQ, McCabe J, et al. Denosumab, a fully human monoclonal antibody to RANKL, inhibits bone resorption and increases $\mathrm{BMD}$ in knock-in mice that express chimeric (murine/human) RANKL, Journal of Bone Mineral Research 2009; 24:182-95.

61. Peddi P, Lopez-Olivo MA, Pratt GF et al. Denosumab in patients with cancer and skeletal metastases: a systematic review and metaanalysis, Cancer Treatment Review 2013; 39:97-04.

62. Heath DJ, Vanderkerken K, Cheng X, et al. An Osteoprotegerin-like Peptidomimetic Inhibits Osteoclastic Bone Resorption and Osteolytic Bone Disease in Myeloma, Cancer Research 2007; 67

63. Kim H, Choi HK, Shin JH, et al., Selective inhibition of RANK blocks osteoclast maturation and function and prevents bone loss in mice, The Journal of Clinical Investigation 2009; 119: 4

64. Téletchéa $S$, Stresing V, Hervouet $S$ et al. Novel RANK Antagonists for the Treatment of Bone-Resorptive Disease: Theoretical Predictions and Experimental Validation, Journal of Bone Mineral Research 2014; 29:1466-77

65. Zhao $\mathrm{Y}$, Jin $\mathrm{M}$, Ma J, et al.,_Inhibition effect of enteropeptidase on RANKL-RANK signalling by cleavage of RANK, FEBS Letters 2013 587:2958-64.

66. Onyia JE, Rachelle J, Galvin RJS, et al. Novel and Selective Small Molecule Stimulators of Osteoprotegerin Expression Inhibit Bone Resorption, Journal of Pharmacology and Experimental Therapeutics 2010; 309:369-79

67. Naidu VG, Dinesh KR, Thwin MM et al. RANKL targeted peptides inhibit osteoclastogenesis and attenuate adjuvant induced arthritis by inhibiting $\mathrm{NF}-\kappa \mathrm{B}$ activation and down regulating inflammatory cytokines, Chemico Biological Interaction 2013; 203:467-79

68. Ma R, Xu J, Dong B, et al. Inhibition of osteoclastogenesis by RNA interference targeting RANK, Musculoskeletal Disorders 2012; 13:154

69. Brennan TC, Rybchyn MS, Green W, et al. Osteoblasts play key roles in the mechanisms of action of strontium ranelate, British Journal of Pharmacology 2009; 157:1291-1300

70. Xiu $\mathrm{Y}, \mathrm{Xu} \mathrm{H}$, Zhao $\mathrm{C}$, et al. Chloroquine reduces osteoclastogenesis in murine osteoporosis by preventing TRAF3 degradation, Joural of Clinical Investigation 2014; 124:297-10

71. Ma X, Liu Y, Zhang Y et al. Jolkinolide B inhibits RANKLinduced osteoclastogenesis by suppressing the activation NF$\kappa \mathrm{B}$ and MAPK signaling pathways, Biochemical ad Biophysical Research Communication 2014; 445:282-8

72. Ha H, Shim K, Kim T, et al. Water Extract of Acer tegmentosum reduces bone destruction by inhibiting osteoclast differentiation and function, Vitamins and Molecules 2014; 19: 3940-54 